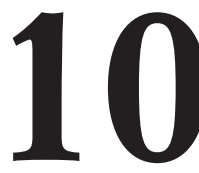

\title{
PARADIGMA ESTRATÉGICO PARA EL DESARROLLO DE HABILIDADES COMPETENCIALES. ESTUDIO DESCRIPTIVO SOBRE LA VARIABILIDAD EN LA PERCEPCIÓN DE HABILIDADES COMPETENCIALES DE 40 ALUMNOS DE EDUCACIÓN FÍSICA EN FASE DE FORMACIÓN INICIAL
}

\section{(STRATEGIC PARADIGM FOR THE DEVELOPMENT OF COMPETENCES SKILLS. DESCRIPTIVE STUDY ON VARIABILITY IN THE PERCEPTION OF COMPETENCES SKILL OF 40 PHYSICAL EDUCATION STUDENTS IN PRESERVICE TRAINING)}

Pere Blanco Felip, M. Carmen Jové Deltell Y Joaquín Reverter Masià

Universidad de Lleida

\section{RESUMEN}

La calidad educativa de los centros de hoy depende del nivel de competencias adquirido por sus educadores (Perrenoud, 2002). En este sentido, debemos apostar por un equipo humano formado por sujetos que sepan superarse permanentemente, aptos para ejercer tareas dinámicas y cambiantes y que compartan alternativas para crecer en todos los sentidos. Más todavía, debemos apostar por profesionales capaces de identificar y dominar los procesos que se deben realizar actuando con decisión y responsabilidad. La adquisición de competencias comporta aptitud y actitud para utilizar conocimientos, más concretamente, habilidades y valores, de manera interdisciplinaria, transversal e interactiva en contextos y situaciones que requieren la intervención de contenidos vinculados a las diferentes áreas del currículum (Lleixà, 2007), sin exclusividad, en este caso, del área de Educación Física. Presentamos una propuesta metodológica, el "Paradigma Estratégico para el desarrollo de habilidades competenciales», cuya ejecución implica comprensión, reflexión y discernimiento, teniendo en cuenta la dimensión social de cada situación.

El estudio se ha llevado a cabo en la Universidad de Lleida con 40 alumnos de tercer curso de la asignatura «Educación Física y su didáctica». Se pretende valorar el impacto que provoca la implementación del programa «Paradigma estratégico para la adquisición de habilidades competenciales» sobre sus propias percepciones competenciales intrapersonales, antes y después de la aplicación de dicho programa. 


\section{ABSTRACT}

The educational quality of schools today depends on the level of competencies acquired by educators. We must focus on the human team, a team made of members that are capable of training and constantly improving their practice, a team that is ready to perform dynamic, changing tasks and whose members share alternatives for resolving all sorts of situations. Even more, they must be able to define and master the processes that they must perform, acting decisively and responsibly, paying attention less to what people might say and more to their own ability to execute, rectify and improve. Acquiring competencies means working on contents, but not exclusively; rather it also means working on the aptitudes and attitudes for using this knowledge, and even more importantly on strategically using skills in an interdisciplinary, transversal and interactive way in different contexts and situations. In this article we present a methodological proposal, namely the Strategic Paradigm for Developing Competency Skills, which ensures attainment of the curricular goals revolving around the skills based on learning how to be and act, learning how to think and communicate, learning how to discover and take initiative, and learning how to peacefully coexist and inhabit the world. Implementation of this model involves understanding, reflection and discernment, bearing in mind the social dimension of each situation not to store more contents but to be strategic and develop competency skills which will consequently increase knowledge, enhance performance, demonstrate attitudes and internalise values.

\section{INTRODUCCIÓN}

Hablar de competencias en Educación Física es hablar de implicación y de compromiso para que dicha educación sea también integral y no se centre exclusivamente en el desarrollo de las capacidades físico-motrices. Ya no se debe pensar en transmitir para acumular o en repetir para mejorar, sino de desarrollar habilidades para lograr, mediante la excusa de la actividad física, la adquisición de aquellas competencias imprescindibles para ser, hacer, aprender y convivir en el mundo (Alberici y Serreri, 2005: 26).

Para que estas competencias tomen cuerpo y resuelvan expectativas, los docentes, también los de Educación Física, tenemos las obligaciones de conocer las directrices que regulan los nuevos sistemas educativos, tanto las políticas educativas como las nuevas propuestas pedagógicas, didácticas y metodológicas. A partir de aquí, debemos preguntarnos qué competencias son necesarias para afrontar con calidad los retos culturales, sociales y laborales en la segunda década del siglo XXI.

Si tomamos como punto de partida el informe Delors (1996), podemos observar que se pone de manifiesto la necesidad de buscar un equilibrio en- 
tre aprender a conocer, hacer, convivir y ser. Para Monereo y Pozo (2007) saber no es simplemente poseer conocimiento, sino también saberlo utilizar. Hacer sin actuar es poner en práctica una acción sin proyectar los sentidos ni encadenar procesos afines, mientras que saber actuar predispone a un conjunto de acciones donde la ejecución de cada una depende del desempeño de la totalidad (Le Boterf, 1994). Saber hacer no es saber imitar, es saber y actuar al mismo tiempo (Tejada, 2005).

Actualmente y con la adaptación de las nuevas titulaciones al espacio europeo de educación superior, tenemos la ocasión de mejorar la educación integral de nuestros alumnos orientando nuestras acciones hacia el desarrollo de habilidades competenciales. El trabajo por competencias provoca un desplazamiento de las intenciones educativas; no se trata de adquirir y sumar conocimientos, sino de ser capaz de ponerlos a prueba en situaciones concretas que lleven a los alumnos hacia el ajuste social mediante un desarrollo de las capacidades personales según afirman Gaskins y Elliot (1999). También afirman que las habilidades sobrevienen de procedimientos aprendidos y automatizados que, posteriormente, se aplicarán de manera inconsciente, produciendo rutinas cognitivas que facilitarán la adquisición y la asimilación del conocimiento. Si estas rutinas se utilizan con eficacia, podremos hablar de destrezas (Riera, 1989; Ruiz, 1994).

Por otra parte, la inclusión de competencias básicas en el currículum escolar pretende que éstas sean alcanzadas mediante objetivos educativos propios de cada área o materias. Como indican diferentes autores, Palacios, Marchesi y Coll (2006), trabajar las competencias en los entornos educativos, facilita la movilización de los conocimientos, aplicarlos en diversas situaciones, poseer un conocimiento funcional y activar los más relevantes para afrontar las situaciones que se van dando en las diversas situaciones del contexto. Si bien en la actualidad el impulso de las competencias está actualizando los programas educativos de vanguardia, es necesario un modelo de intervención que indique un itinerario claro para la adquisición de habilidades competenciales. Sin una propuesta para que los docentes se impliquen de forma efectiva y activa en el conocimiento y desarrollo de habilidades, es lógico pensar que la adquisición de las competencias no será efectiva si no se integra el saber, saber hacer y saber ser (Bisquerra Alzina 2003:22). En este sentido Blanco (2007) considera que para adelantar el logro de las competencias, es fundamental enmarcar los procesos de enseñanza-aprendizaje alrededor de cuatro ejes: el primero de ellos, el contexto, que ofrece la oportunidad de vivenciar valores democráticos y solidarios; el segundo, la experiencia, que aporta el placer de disfrutar del conocimiento; el tercer eje, la reflexión, que facilitará la adquisición de una actitud crítica y, por último, la acción, que procura la autonomía. 
Diversos autores preceden, con sus estudios previos sobre las habilidades, su clasificación y desarrollo, nuestra investigación (Meinel y Schnabel, 1987; Monereo, 1994; Manno, 1995; Goleman, 1995; Tedesco, 1997; Gardner, 1998; Agudelo, 2002; Bisquerra 2003).

Es a partir de sus aportaciones que concretamos nuestra propuesta a partir del Paradigma Estratégico (Blanco, 2010). Resultado ecléctico entre las etapas sucesivas y simultáneas del Paradigma Pedagógico Ignaciano (Blanco, 2004; CONEDSI, 1993; Reyes, 2008), y la integración de las fases de la Investigación-Acción (Lewin, 1946), con las que se fusionan afectando e interactuando con las habilidades competenciales. El Paradigma Estratégico no se refiere exclusivamente al proceso de transmisión de contenidos, incluye también la relación educador - educando y las dinámicas que lo soportan, de lo contrario, podría darse el peligro de alejarse de la adquisición de las competencias que es, en definitiva, lo que se pretende lograr. Todo el proceso educa, el Paradigma está al servicio de la gestión de las competencias y no sólo la del conocimiento pone énfasis en la interacción continua entre la experiencia, la reflexión y la acción como medio de autoevaluación del aprendizaje de las competencias: cómo se adquieren y desarrollan en estrecha correspondencia durante cinco etapas: Contextualizar el aprendizaje; experimentarlo vivencialmente; reflexionar sobre esa experiencia; actuar consecuentemente y evaluar la acción y el proceso llevado a cabo.

Bajo estos presupuestos, el estudio que presentamos pretende comprobar los efectos que el Paradigma Estratégico para el Desarrollo de Habilidades Competenciales aplicado sobre un grupo de futuros docentes, provoca en la propia percepción de las habilidades que se consideran básicas para trabajar en la formación inicial de los maestros.

\section{METODOLOGÍA}

Nuestra propuesta metodológica establece una relación entre diferentes métodos que fusionan los soportes éticos, teóricos, organizativos, humanos y técnicos para dar respuesta a los cambios, bajo conceptos de contexto y realidad, experiencia y conocimiento, reflexión y acción y, evaluación y comunicación. Se trata, en definitiva, de generar un comportamiento estratégico válido que sea útil para el desarrollo de las habilidades competenciales. Las metodologías no tienen que preceder a las necesidades y objetivos de los educadores, sino que han de ser estos los que busquen las técnicas más apropiadas para el desarrollo de habilidades. En esta línea, defendemos las ventajas de un cierto eclecticismo, ya que facilita el aprovechamiento de distintas alternativas metodológicas. Una visión ecléctica, con la integración de los procesos cíclicos de la Investigación-Acción (Lewin, 1946); las bases 
fundamentales del Paradigma Pedagógico Ignaciano (Blanco, 2004; CONEDSI, 1993; Reyes, 2008); la dinámica cooperativa del Rompecabezas que proponen Aronson y Patnoe (1997) y Walters (2000), junto a diversas técnicas que favorecen el trabajo cooperativo (Slavin,1999).

\subsection{Muestra}

El estudio se realizó con 40 alumnos del último curso de la titulación de maestro de primaria, de la facultad de Ciencias de la Educación de la Universidad de Lleida. Todos los alumnos participaban de la asignatura troncal Educación Física y su didáctica, con una carga crediticia de 4,5 créditos.

\subsection{Instrumento}

El cuestionario utilizado tipo escala de actitudes Likert es de diseño propio, basado en un estudio realizado por Blanco (2010). Dicho cuestionario fue creado por expertos en el diseño de cuestionarios. Fue validado y testado por criterio de jueces competentes y calificados. Se trataba de averiguar si los jueces estaban de acuerdo o no en que los ítems medían habilidades. Se realizó, también, un estudio piloto con grupos de alumnos de la titulación de maestros de la Facultad de Ciencias de la Educación. El cuestionario está dividido en cuatro dominios necesarios para determinar con mayor objetividad el grado de estimación de los sujetos para cada una de las diferentes habilidades: (a) habilidades cognitivas-intelectuales, (b) habilidades físicomotrices, (c) habilidades sociales-comunicativas, (d) habilidades intrapersonales. Consta de cuarenta y ocho ítems en los que se debe anotar el grado de acuerdo o desacuerdo con las afirmaciones contenidas en los cuatro dominios. Para la escala de estimación se consideró la cifra de cinco como la más adecuada ya que centraba más las respuestas.

\subsection{Diseño}

Se utilizó un diseño longitudinal cuasiexperimental con medición pretest/post-test, con la finalidad de que se pudiera contestar sin la ayuda del investigador (Salkind,1999). Se pretende obtener información de la situación en la que se encuentran los futuros docentes antes de utilizar el Paradigma Estratégico. Mostramos, a continuación, una tabla que recoge el instrumento utilizado para cada fase de la investigación, así como el compilador. 


\begin{tabular}{|c|c|c|}
\hline FASE & INSTRUMENTO & COMPILADOR \\
\hline Previa & Cuestionario abierto & Alumnos \\
\hline Implementación & Paradigma estratégico & Profesor/Alumnos \\
\hline \multirow{2}{*}{ Posterior } & Cuestionario abierto & Alumnos \\
\cline { 2 - 3 } & Paquete estadístico & Profesor \\
\hline
\end{tabular}

Tabla 1. Instrumentos utilizados en las distintas fases del estudio

El Paradigma aplicado estuvo dividido en las cuatro dimensiones que nos propone el Paradigma Pedagógico (CONEDSI, 1993), con una duración de 15 horas para cada una de ellas. Los contenidos de las dimensiones fueron los siguientes:

Dimensión 1: Contexto con el grupo propio. Contribuye significativamente al desarrollo sin perder de vista el entorno, el ambiente y los conocimientos previos adquiridos con anterioridad.

Dimensión 2: Experiencia con el grupo de expertos. La experiencia convierte el proceso de enseñanza y de aprendizaje en una acción intencional con propósitos definidos y establece las relaciones entre los diferentes protagonistas.

Dimensión 3: Reflexión con el grupo propio. La reflexión parte del conocimiento, no es fruto de la curiosidad, sino que más bien responde a la necesidad de discernimiento de manera atenta y crítica y, a partir de la resolución de problemas se puede trabajar de manera más crítica para avanzar en los procesos didácticos.

Dimensión 4: Acción con el grupo general de la clase. La persona no es solamente razón o sentimientos, no es solamente ideas. La persona también es acción. A la acción se adhieren aquellos factores que a través de la experiencia adquirida y precedida de su contexto se unen a la reflexión. Todo aquello planificado y asentido ya puede implementarse favoreciendo el desarrollo de pensamientos, de conductas y de alternativas incompatibles con los puntos débiles.

En cada una de las dimensiones se aplicó, en espiral, el proceso de investigación-acción: planificación, acción, observación y reflexión (Lewin, 1946). 
Tras la finalización de dicho Paradigma de intervención, se procedió a obtener las mediciones post-test mediante el mismo cuestionario de evaluación aplicado en la fase pre-test.

Ofrecemos, a continuación, una tabla que resume los aspectos más importantes de dicho paradigma: estructura, dimensiones y técnicas utilizadas.

\begin{tabular}{|c|c|c|c|c|}
\hline \multicolumn{4}{|c|}{$\begin{array}{c}\text { PARADIGMA ESTRATÉGICO PARA EL DESARROLLO } \\
\text { DE HABILIDADES COMPETENCIALES }\end{array}$} \\
\hline $\begin{array}{c}\text { DIMENSIONES } \\
\text { PPI }\end{array}$ & CONTEXTO & EXPERIENCIA & REFLEXIÓN & ACCIÓN \\
\cline { 2 - 5 } & Grupo propio & Grupo expertos & Grupo propio & Grupo curso \\
\hline
\end{tabular}

\begin{tabular}{|c|c|c|c|c|}
\hline $\begin{array}{l}\text { ROMPE- } \\
\text { CABEZAS }\end{array}$ & $\begin{array}{l}\text { Presentación } \\
\text { del tema, in- } \\
\text { tenciones y } \\
\text { objetivos. } \\
\text { Concreción de } \\
\text { los contenidos } \\
\text { de trabajo a } \\
\text { desarrollar. } \\
\text { Concreción de } \\
\text { dudas, concep- } \\
\text { tos, situacio- } \\
\text { nes. } \\
\text { Distribución } \\
\text { de los conteni- } \\
\text { dos del tema a } \\
\text { los miembros } \\
\text { del grupo para } \\
\text { su desarrollo } \\
\text { personal. }\end{array}$ & $\begin{array}{l}\text { Búsqueda in- } \\
\text { dividualmente } \\
\text { de experien- } \\
\text { cias directas e } \\
\text { indirectas. } \\
\text { Presentación } \\
\text { de evidencias } \\
\text { sobre los con- } \\
\text { tenidos a desa- } \\
\text { rrollar: qué, } \\
\text { quién, dónde, } \\
\text { cuándo, cómo. } \\
\text { Búsqueda de } \\
\text { relaciones con } \\
\text { otros conteni- } \\
\text { dos o temas } \\
\text { que desarro- } \\
\text { llan los demás } \\
\text { miembros }\end{array}$ & $\begin{array}{l}\text { Presentar al } \\
\text { grupo propio } \\
\text { la experiencia } \\
\text { desarrollada. } \\
\text { Contraste y } \\
\text { unificación de } \\
\text { la informa- } \\
\text { ción. } \\
\text { Concreción de } \\
\text { actividades } \\
\text { que puedan } \\
\text { reforzar el } \\
\text { aprendizaje } \\
\text { Valoración y } \\
\text { corrección de } \\
\text { aspectos for- } \\
\text { males } \\
\text { Valoración del } \\
\text { grupo y de las } \\
\text { tareas realiza- } \\
\text { das individual- } \\
\text { mente. }\end{array}$ & $\begin{array}{l}\text { Distribución y } \\
\text { temporaliza- } \\
\text { ción de la pre- } \\
\text { sentación. } \\
\text { Presentación } \\
\text { individual de } \\
\text { contenidos por } \\
\text { cada grupo } \\
\text { propio utili- } \\
\text { zando nuevas } \\
\text { tecnologías de } \\
\text { la informa- } \\
\text { ción. } \\
\text { Realización de } \\
\text { actividades } \\
\text { previstas en } \\
\text { los distintos } \\
\text { temas. } \\
\text { Presentación } \\
\text { del trabajo de- } \\
\text { finitivo al pro- } \\
\text { fesor. }\end{array}$ \\
\hline
\end{tabular}




\begin{tabular}{|c|c|c|c|c|}
\hline \multicolumn{5}{|c|}{ TÈCNICAS ESTRATÉGICAS } \\
\hline $\begin{array}{c}\text { INVESTIGA- } \\
\text { CIÓN } \\
\text { ACCION }\end{array}$ & $\begin{array}{c}\text { DIAGRAMA } \\
\text { DE ISHIKAWA }\end{array}$ & $\begin{array}{c}6 \\
\text { PREGUNTAS }\end{array}$ & DAFO & SIMPOSIUM \\
\hline $\begin{array}{l}\text { PLANIFICA- } \\
\text { CIÓN }\end{array}$ & $\begin{array}{l}\text { Definición y } \\
\text { clasificación } \\
\text { de los conte- } \\
\text { nidos de tra- } \\
\text { bajo a desa- } \\
\text { rrollar. }\end{array}$ & $\begin{array}{l}\text { Organización } \\
\text { de la búsque- } \\
\text { da de infor- } \\
\text { mación: Téc- } \\
\text { nica de las } 6 \\
\text { preguntas. }\end{array}$ & $\begin{array}{l}\text { Aporte de la } \\
\text { experiencia } \\
\text { adquirida me- } \\
\text { diante la bús- } \\
\text { queda de in- } \\
\text { formación. }\end{array}$ & $\begin{array}{l}\text { Temporizar la } \\
\text { presentación. }\end{array}$ \\
\hline ACCIÓN & $\begin{array}{l}\text { Distribución } \\
\text { de los conte- } \\
\text { nidos del } \\
\text { tema median- } \\
\text { te un diagra- } \\
\text { ma de Ishika- } \\
\text { wa. }\end{array}$ & $\begin{array}{l}\text { Elaboración } \\
\text { de fichas de } \\
\text { lectura con } \\
\text { palabras cla- } \\
\text { ve, ideas fun- } \\
\text { damentales y } \\
\text { resumen de } \\
\text { texto. }\end{array}$ & $\begin{array}{l}\text { Presentación } \\
\text { de puntos dé- } \\
\text { biles y fuertes, } \\
\text { amenazas y } \\
\text { fortalezas de } \\
\text { los conteni- } \\
\text { dos. }\end{array}$ & $\begin{array}{l}\text { Presentación } \\
\text { oral y física } \\
\text { por parte de } \\
\text { todos los } \\
\text { miembros de } \\
\text { cada grupo } \\
\text { del tema desa- } \\
\text { rrollado. }\end{array}$ \\
\hline $\begin{array}{l}\text { OBSERVA- } \\
\text { CIÓN }\end{array}$ & $\begin{array}{l}\text { La significa- } \\
\text { ción de los } \\
\text { contenidos } \\
\text { trabajados. }\end{array}$ & $\begin{array}{l}\text { Que la infor- } \\
\text { mación reco- } \\
\text { gida sea útil, } \\
\text { actual y pueda } \\
\text { reverenciarse. }\end{array}$ & $\begin{array}{l}\text { Confección de } \\
\text { un diario re- } \\
\text { flexivo }\end{array}$ & $\begin{array}{l}\text { Valoración y } \\
\text { evaluación del } \\
\text { trabajo reali- } \\
\text { zado por cada } \\
\text { grupo. }\end{array}$ \\
\hline REFLEXIÓN & $\begin{array}{l}\text { Relación de } \\
\text { los contenidos } \\
\text { de aprendiza- } \\
\text { je con las ha- } \\
\text { bilidades sig- } \\
\text { nificativas. }\end{array}$ & $\begin{array}{l}\text { Aporte de ex- } \\
\text { periencias } \\
\text { personales, } \\
\text { opiniones y/o } \\
\text { comentarios } \\
\text { en las fichas. }\end{array}$ & $\begin{array}{l}\quad \text { Valora- } \\
\text { ción y correc- } \\
\text { ción utilizan- } \\
\text { do la técnica } \\
\text { de valoración } \\
\text { de iguales }\end{array}$ & $\begin{array}{l}\text { Ejecución de } \\
\text { las actividades } \\
\text { de aprendiza- } \\
\text { je propuestas } \\
\text { por los dife- } \\
\text { rentes grupos. }\end{array}$ \\
\hline
\end{tabular}

Tabla 2. Dimensiones del Paradigma Estratégico

\section{RESULTADOS}

Para valorar los resultados obtenidos, ofrecemos a continuación un cuadro-resumen que aglutina las diferentes categorías y subcategorías que han considerado nuestros alumnos en el momento de completar el cuestionario. Dicho cuestionario se ha completado teniendo en cuenta, en primer 
lugar, la pregunta "qué habilidades crees que tienes para ser un buen maestro» y, en segundo lugar, los dos momentos en que se completa el cuestionario -pre-test y post-test-.

\begin{tabular}{|c|c|}
\hline CATEGORIA & SUBCATEGORIA \\
\hline \multirow{5}{*}{ HABILIDADES INTERPERSONALES } & EMPATÍA \\
\hline & INFLUENCIA \\
\hline & COMUNICACIÓN \\
\hline & MANEJO CONFLICTOS \\
\hline & COLABORACIÓN Y COOPERACIÓN \\
\hline \multirow{9}{*}{ HABILIDADES INTRAPERSONALES } & AUTOVALORACIÓN \\
\hline & AUTOCONTROL \\
\hline & CONFIABILIDAD \\
\hline & CONCIENCIA \\
\hline & ADAPTABILIDAD \\
\hline & INNOVACIÓN \\
\hline & COMPROMISO \\
\hline & INICIATIVA \\
\hline & OPTIMISMO \\
\hline \multirow{6}{*}{ HABILIDADES COGNITIVAS } & OBSERVACIÓN DE LOS FENÓMENOS \\
\hline & ORDEN DE LOS HECHOS \\
\hline & MEMORIZACIÓN \\
\hline & INTERPRETACIÓN \\
\hline & TRANSFERENCIA \\
\hline & VALORACIÓN DE LOS APRENDIZAJES \\
\hline \multirow{4}{*}{ OTRAS } & SEPARAR TAREAS DE CASA/TRABAJO \\
\hline & BUENA IMAGEN \\
\hline & PERSONA PRÁCTICA \\
\hline & ATENDER NNEE \\
\hline
\end{tabular}

Tabla 3. Relación de categorías y subcategorías

El gráfico que ofrecemos a continuación muestra la relación por categorías en los dos momentos de aplicación del cuestionario pre-test y post-test: 


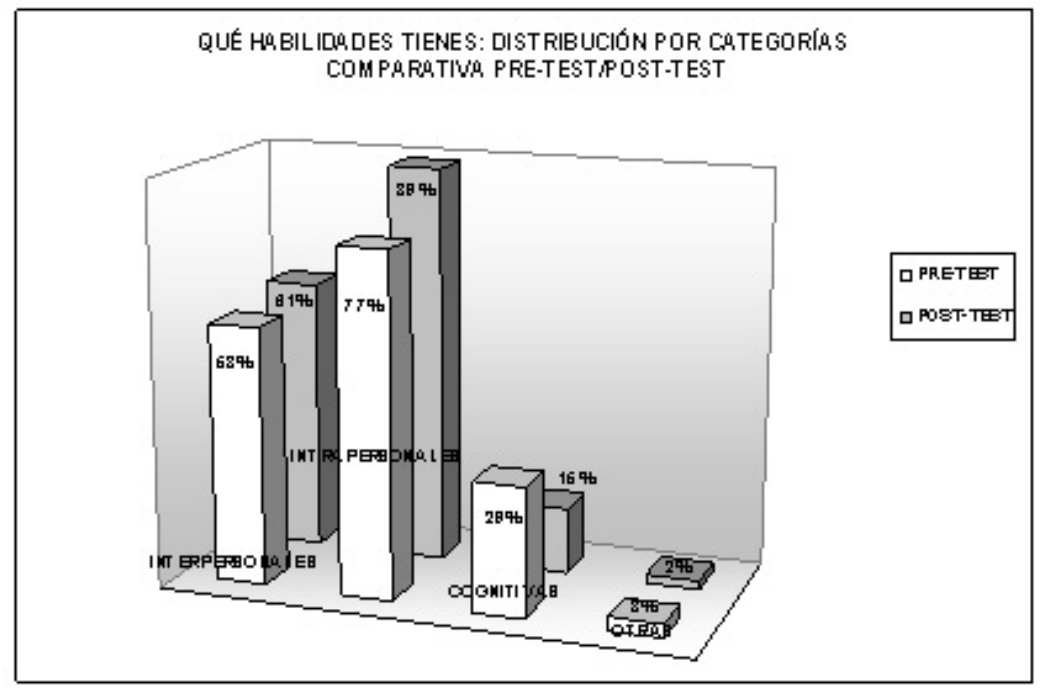

Gráfico 1. Relación por categorías -pre-test/post-test-

Si atendemos en primer lugar a la distribución por categorías, podemos observar cómo la relación entre ellas varía si consideramos los distintos momentos de implementación del cuestionario. De esta manera, las dos primeras categorías -habilidades sociales interpersonales y habilidades sociales intrapersonales- incrementan su presencia en el post-test, mientras que las habilidades cognitivas y las que pertenecen a la categoría "otras», descienden su presencialidad siendo más significativa en la categoría de las habilidades cognitivas. Se evidencia, pues, un incremento importante en la categoría habilidades intrapersonales entre el pre-test (77\%) y el post-test (89\%), así como un descenso considerable en la categoría de habilidades cognitivas, que pasa de $29 \%$ en el pre-test a un $15 \%$ en el post-test.

La siguiente tabla — habilidades sociales interpersonales-, muestra la relación entre las diferentes subcategorías, antes y después de la implementación del programa: 


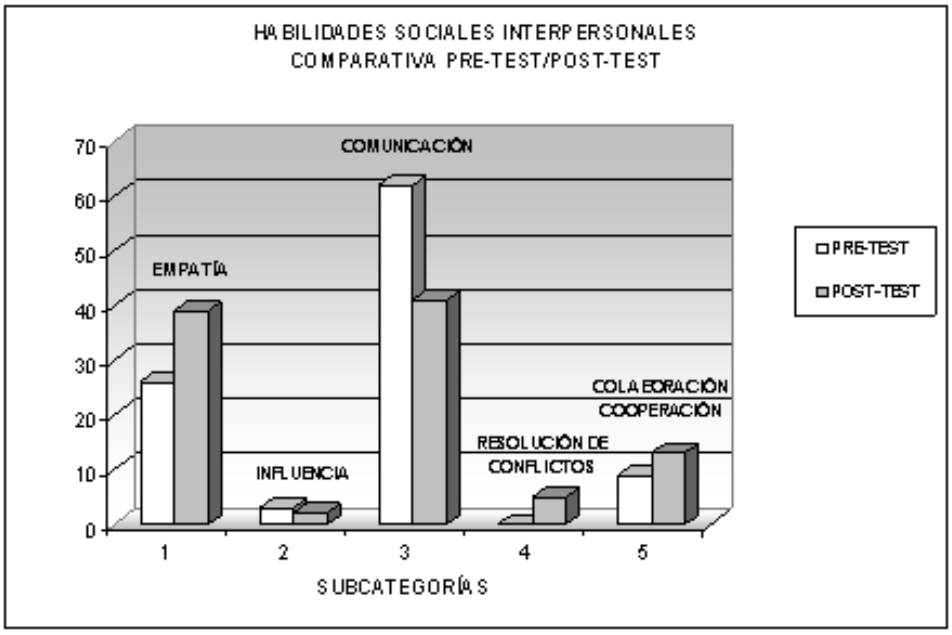

Gráfico 2. Habilidades sociales interpersonales.

Podemos observar cómo la «empatía», la «resolución de conflictos» y la «colaboración-cooperación» tienden a aumentar en el post-test, mientras que la «influencia» y la "comunicación» evidencian un notable retroceso en el post -test.

Nos referimos ahora a la categoría «habilidades intrapersonales» y nos detenemos en la relación que guardan entre si las diferentes subcategorías en los dos momentos de implementación del cuestionario.

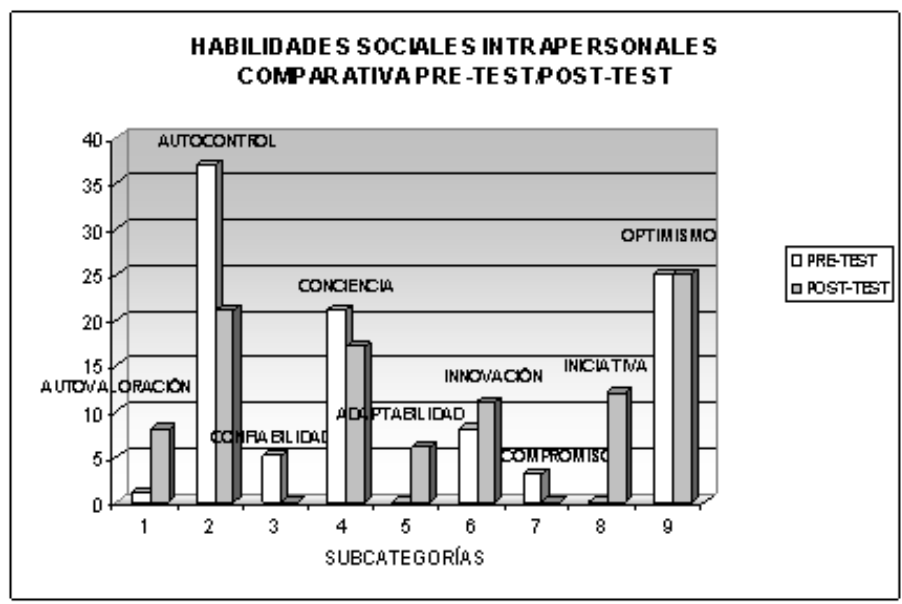

Gráfico 3. Habilidades sociales intrapersonales. 
Podemos observar como, si en el pre-test nuestros alumnos creen poseer autocontrol, confiabilidad y conciencia, en el post-test, no sienten que posean dichas habilidades ya que su presencia en porcentajes disminuye, sobre todo, para la habilidad de autocontrol, que pasa del 37\% en el pre-test al $21 \%$ en el post-test.

En cuanto a las habilidades cognitivas, podemos observar cómo en el pre-test, nuestros alumnos, sobre todo, se consideran competentes en observar los fenómenos -31\%-, y en ordenar los hechos -28\%-. Es significativo cómo, en el post-test, estas habilidades pierden importancia considerablemente para dar paso a un incremento en las habilidades de interpretación $20 \%$ - y transferencia $-41 \%$ - .

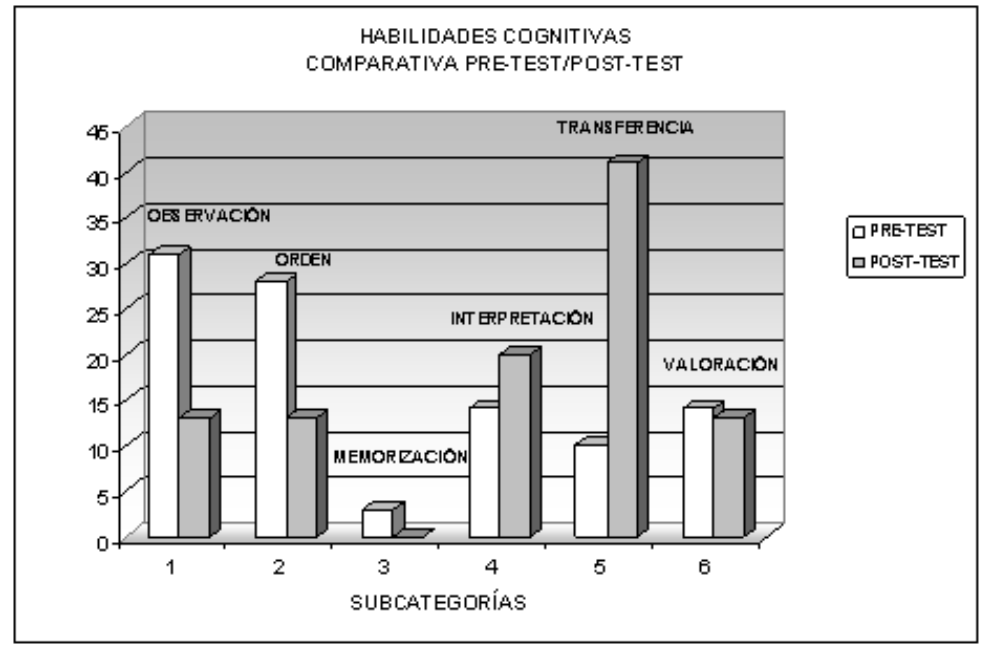

Gráfico 4. Habilidades cognitivas.

\section{DISCUSIÓN}

El estudio constata la posibilidad de modificar las habilidades interpersonales dentro del aula. En un primer momento de la investigación -pretest-, nuestros alumnos ponen de manifiesto que son poco empáticos (26\%), que tienen nula capacidad para resolver conflictos $(0 \%)$ y que se reconocen con poca habilidad para colaborar con los demás (9\%). En un segundo momento de la investigación -post-test-, se consideran más empáticos (39\%), mejor preparados para resolver conflictos $(5 \%)$ y con mayor capacidad para colaborar y cooperar (13\%). Por lo tanto, se mejora la capacidad de aprender a compartir juntos, poniéndose en el lugar de los otros, sin exclusiones y res- 
petando la diversidad (Salovey y Sluyter, 1997). También se abordan abiertamente las cuestiones que presentan mayor discusión y controversia, asumiendo un discurso dialogante en la línea de Casel (2006), para resolver situaciones gracias a la transferencia e interpretación de los fenómenos (Echeverría, 2005), compartiendo proyectos, informaciones y recursos a través del clima cooperativo (Monereo, 2007).

En cuanto a la capacidad de influencia y a la habilidad de relacionarse entre ellos, destacar que, antes de iniciar el programa -pre-test-, nuestros alumnos consideraron poseer una buena capacidad de comunicación e influencia ( $62 \%$ y $3 \%$, respectivamente), mientras que, una vez finalizado el programa -post-test-, manifestaron no poseer dichas habilidades en la misma proporción -comunicación $41 \%$; influencia 3\%-. A este respecto podemos considerar que las habilidades interpersonales favorecen la interacción del proceso de aprendizaje generando sinergias que disminuyen la intensidad de la comunicación (Medina, 2006), al mismo tiempo que favorece el desarrollo de habilidades intrapersonales.

Analizando las habilidades intrapersonales, resulta evidente que aquellas que más incrementan su presencia en el post-test son la autovaloración, la adaptabilidad, la innovación y la iniciativa. Resaltamos en este momento la importancia de la aplicación del programa ya que tiende a optimizar habilidades que nos preparan para adaptarnos a diferentes situaciones y a tomar la iniciativa para mejorar o innovar distintos aspectos relacionados con la educación. En este sentido, se facilita un mayor autocontrol, habilidad imprescindible para establecer la regulación reflexiva de las emociones y promover el crecimiento personal (Saarni, 2000), al mismo tiempo que se genera cierto optimismo, habilidad indispensable que permite afrontar los retos diarios con intención de ser justos y compasivos (Sternberg, 1997), reforzando la franqueza y la fortaleza de uno mismo (Chernis, 2000). Por lo que respecta a la mejora en innovación, hemos podido observar que se asumen mayores riesgos en las decisiones (Muñoz-Nájar, 2002). Queremos destacar las subcategorías «adaptabilidad» e «iniciativa», ya que en un primer momento -pre-test-, casi no se habían considerado, mientras que en el post-test sí las destacan y valoran.

Chernis (2000), considera que el aumento de habilidades intrapersonales mejora las habilidades cognitivas, ampliamente desarrolladas en etapas educativas anteriores, generando una mayor estabilidad personal. Se puede contrastar con los resultados obtenidos en las habilidades cognitivas. Los alumnos valoran una concepción del aprendizaje mucho más constructivista en la que su funcionalidad parece ser el factor al que más importancia se le da. En un primer momento -pre-test-, no consideran importante poseer la habilidad de transferir los aprendizajes a otros ámbitos del proceso de en- 
señanza-aprendizaje, o lo que es lo mismo, a otros aspectos de la vida cotidiana, mientras que, después de la aplicación del programa -post-test-, consideran en un $41 \%$ que sí poseen tal habilidad. En este sentido, la utilización de estrategias más participativas y colaborativas en la formación inicial de los maestros, como es el caso del programa de aplicación, favorece una visión más global, práctica y funcional del propio proceso de enseñanza-aprendizaje, acentuando de manera especial la acción reflexiva. De igual manera sucede con la habilidad de interpretación: incrementa su presencia en el post-test, pasando del $14 \%$ al 20\%. Destacamos, también, la habilidad "memorización», ya que en el pre-test se le otorga una mínima presencia -3\%-y, en el post-test, desaparece por completo -0\%-.

\section{CONCLUSIONES}

Podemos afirmar que la propuesta que presentamos «Paradigma Estratégico para la adquisición de habilidades competenciales», determina una necesidad de cambio en nuestros planteamientos iniciales. La adquisición de unas determinadas habilidades depende, enormemente, de la actuación metodológica que se desarrolle durante el proceso de aprendizaje. No son los contenidos quienes influyen sustancialmente en el proceso sino la forma de trabajarlos.

Con respecto a la estrategia utilizada, ésta ha permitido a los alumnos tomar conciencia de sus habilidades y, a la vez, de sus limitaciones. En este sentido, la implementación del programa ha potenciado: a) La empatía para darse cuenta de los sentimientos, sensibilidades, opciones y perspectivas intelectuales de las personas que nos rodean; b) La comunicación para ser capaces de dialogar asertivamente, ser claros y comprensibles mediante el uso de la escucha y la transmisión de los mensajes. Hablar cuando es el momento, escuchar sin ironías y evitar las intimidaciones y, c) la cooperación, atendiendo a la necesidad de trabajar con los demás para conseguir objetivos interpersonales.

Asímismo a nivel personal el programa genera mejoras en: a) El autocontrol como habilidad que nos permite ser receptivos a los sentimientos, ya sean agradables o desagradables, y a resistir los impulsos para actuar y controlar tensiones y emociones; b) El optimismo como habilidad para automotivarse, ver el aspecto más brillante de nuestra vida y tener una actitud positiva; c) La conciencia para saber asumir responsabilidades de los propios actos como regulación de la propia persona, desarrollando la identidad intrapersonal y, d) La innovación, como habilidad que permite cierta actitud de apertura y disposición en el diseño y la aplicación de acuerdos creativos y novedosos, evitando responder por defecto de la misma forma en situaciones 
y problemas que requieren soluciones no convencionales. En este sentido, la implementación del programa favorece en los alumnos la transferencia y la interpretación, habilidades que logran aplicar los conocimientos asimilados en procesos anteriores, a situaciones nuevas y a contextos distintos. En la misma línea, se favorece la observación para identificar aquellos elementos aislados de un todo, o del todo. Conseguir el desarrollo de esta habilidad cognoscitiva es requisito indispensable para acceder a otras. También incide sobre el orden de los hechos, es decir, la disposición sistemática de un conjunto de datos a partir de un atributo concreto, eso supone saber reunir, agrupar y listar series y encadenamientos. Siguiendo con el mismo discurso, la aplicación del programa orienta a nuestros alumnos hacia una concepción del aprendizaje mucho más constructivista en la que su funcionalidad parece ser un factor de gran importancia.

Hemos de plantear nuevos diseños de actuaciones curriculares que identifiquen plenamente las competencias profesionales que han de adquirir nuestros alumnos antes de certificar su formación académica, como el constructo molar que define Zabalza (2009) refiriéndose al conjunto de conocimientos, habilidades y estrategias que necesitamos para desarrollar una actividad. A la vez, tales diseños deben desarrollar estrategias que favorezcan de manera integral la adquisición de habilidades competenciales gracias al predominio estratégico de técnicas y procedimientos en coherencia con los diferentes dominios: intrapersonal, interpersonal, cognitivo y físico-motriz, basados en la reflexión que promulgan, por ejemplo, Lago, 2007; Onorrubia 2004. Los nuevos diseños de actuaciones curriculares deben tender hacia la adquisición de habilidades intrapersonales por encima de las habilidades cognitivas, ampliamente desarrolladas en etapas educativas anteriores. La adquisición de las mismas genera una estabilidad personal que repercutirá en la calidad profesional que deseamos en nuestros docentes. 


\section{REFERENCIAS BIBLIOGRÁFICAS}

Agudelo, S. (2002). Alianza entre formación y competencia. Reminiscencias de una vida profesional. Montevideo: Cintefor/OIT.

Alberici, A.; Serreri, P. (2005). Competencias y formación en la edad adulta. Balance de competencias. Barcelona: Alertes.

Aronson, E.; Patnoe, S. (1997). The jigsaw classroom: Building cooperation in the classroom (2nd ed.). New York: Addison Wesley Longman.

Bisquerra, R. (2003). Educación emocional y competencias básicas para la vida. Revista de Investigación Educativa (RIE), 21 (1), 7-43.

Blanco, P. (2004). Equips cooperatius, cultura democrática i formació contínua, competències per a una transformació adequada dels centres educatius de secundària. Universidad de Lleida: Tesis inédita.

Blanco, P. (2007). Un marc vivencial per al desenvolupament integral de les competències. Lleida: Edicions Universitat de Lleida.

Blanco, P. (2008). El trabajo cooperativo: una competencia bàsica para la transformación de los centros de secundaria. Revista Iberoamericana de Educación, 46, 11-16.

Blanco, P. (2009). Adquirir competencias desde las habilidades competenciales. Revista Aula de Encuentro, 12, 55-174.

Bisquerra Alzina, R. (2003). Educación emocional y competencias básicas para la vida. Revista de Investigación Educativa, 21 (1), 7-43.

Blanco, P. (2010). Docents innovadors $i$ estratègicament competents. Lleida: $\mathrm{Pa}-$ perkite.
Collaborative for Academic, Social, and Emotional Learning (2006). SEL Competences. University of Illinois at Chicago. Disponible en: http://www.casel.org/about_sel/SELskills.php [Consulta 2006, 15 de noviembre].

CONEDSI, Comisión Nacional de educación. (1993). Pedagogía Ignaciana. Un planteamiento Práctico. Madrid: CONEDSI.

Cherniss, C. (2000). Social and Emotional Competence in the Workplace, in Bar-On, R.; Parker, J. D. The Handbook of Emotional Intelligence. Theory, Development, Assessment, and Application at Home, School, and in the Workplace. San Francisco, Ca: Jossey-Bass, 433-459.

Delors, J. (1996). La educación encierra un tesoro. Informe UNESCO. Madrid: Santillana/UNESCO.

Echeverria, B. (2005). Competencia de acción de los profesionales de la orientación. Madrid: Esic Editorial.

Gardner, H. (1998). Inteligencias múltiples. Barcelona: Paidós.

Gaskins, I.; Elliot, T. (1999). Cómo enseñar estrategias cognitivas en la escuela. Barcelona: Paidós

Goleman, D. (1995). La inteligencia emocional. Barcelona: Kairós.

Jové, M. C. (2005). Model d'intervenció psicomotriu vivenciada per a la prevenció del risc social en educació infantil. Tesis doctoral dirigida por el Dr. Carles Alsinet. Universitat de Lleida. Inèdita.

Lago, J.R. (2007). L'assessorament educatiu com a eina per a la reflexió i la pràcticaeducativa i de l'escola inclussiva. Suports, 11 (1), 42-50. 
Le Boterf, G. (1994). De la competence: essai sur un attracteur étrange. París: Les Editions d'Organisation.

Lewin, K. (1946). Action research and minority problems. Journal of Social Issues, 2 (4), 34-46.

Lleixà, T. (2007). Educación Física y competencias Básicas. Contribución del área a la adquisición de las competencias básicas del currículo. Tandem, $23,31-37$.

Manno, R. (1995). Fundamentos del entrenamiento deportivo. Barcelona: Paidotribo.

Medina, A. M.; Domínguez, M.G. (2006). Los procesos de observación del pràcticum: análisis de las competencias. Revista española de pedagogía, 233, 69103.

Monereo, C. (1994). Estrategias de enseñar y aprendizaje. Formación del profesorado y aplicación en la escuela. Barcelona: Graó.

Monereo, C.; Pozo, J. (2007). Competencias para (con) vivir con el siglo XXI. Cuadernos de Pedagogía, 370, 12-18.

Muñóz-Nájar, J.A.; Vilá, J. (2002). El sistema de innovación: competencias organizativas y directivas para innovar. Revista Dyna, 8, 13-21.

Onrubia, J. (Coord); Fillat, M.; Martínez, $M^{\mathrm{a}}$ D. y Udina, M. (2004). Criterios y recursos para la atención a la diversidad en secundaria. Barcelona: Graó.

Palacios, J.; Marchesi, A. y Coll, C. (2006). Desarrollo psicológico y Educación (vol. I, II). Madrid: Alianza.

Perrenoud, Ph. (2002). D’une métaphore à l'autre: transférer ou mobiliser ses connaissances? En J. Dolz et E. Ollagnier (Eds.) L'énigme de la compétence en éducation. Bruselas: Éditions De Boeck Université, 45-60.
Reyes, F. (2008). La reflexión de la dinámica del Paradigma Pedagógico Ignaciano. Revista Académica, 4, 46- 52.

Riera, J. (1989). Fundamentos del aprendizaje de la técnica y la táctica deportivas. Barcelona, INDE.

Ruiz, L. M. (1994). Deporte y aprendizaje. Procesos de adquisición y desarrollo de habilidades. Madrid: Visor Distribuciones.

Saarni, C. (2000). Emotional Competence. A Developmental Perspective, in Bar-On, R. y Parker J.D. (Eds.), The Handbook of Emotional intelligence.Theory, Development, Assessment, and Application at Home, School, and in the Workplac. San Francisco, Ca: Jossey-Bass, 68-91.

Salkind, N.J. (1999). Métodos de investigación. México: Prentice Hall.

Salovey, P.; Sluyter, D. J. (1997). Emotional Development and Emotional Intelligence. Educational Implications. Nueva York: Basic Books.

Slavin, R.E. (1999). Aprendizaje cooperativo: teoría, investigación y práctica. Buenos Aires: Prentice Hall.

Sternberg, R. (1997). Inteligencia exitosa: Cómo una inteligencia práctica y creativa determinan el éxito en la vida. Barcelona: Paidós.

Tedesco, J.C. (1997). Educación moral, en Marina, J. A. El problema moral de nuestro tiempo. Murcia: Cajamurciana.

Walters, L. S. (2000). Four Leading Models, Harvard Education Letter's Research. Disponible en: http://www.edletter.org/past/issues/2000mj/models.shtm 1 [consulta: 2010, 9 de septiembre].

Zabalza, M. A. (2009). Competencias docentes del profesorado universitario. $\mathrm{Ca}$ lidad y desarrollo profesional. Madrid: Narcea 


\section{PALABRAS CLAVE}

Competencias educativas, habilidades competenciales, Paradigma Estratégico.

\section{KEY WORDS}

Professional competencies, competency skills, strategic paradigm, strategic learning.

\section{PERFIL ACADÉMICO Y PROFESIONAL DE LOS AUTORES}

Miembros del área de Expresión Corporal del Departamento de Didácticas específicas de la Universidad de Lleida. Miembros del grupo de investigación "Movimiento humano», cuyas líneas principales son: a) Actividad física y salud; b) Desarrollo de competencias y, c) Intervención psicomotriz en colectivos de riesgo.

Dirección de les autores: Departamento de Didácticas Específicas Facultad de Ciencias de la Educación Universidad de Lleida Av. de l'Estudi General, 4. 25001 Lleida E-mail: pere.blanco@didesp.udl.cat carme.jove@didesp.udl.cat reverter@didesp.udl.cat

Fecha Recepción del Artículo: 03. Noviembre. 2010

Fecha Revisión del Artículo: 10. Abril. 2011

Fecha Aceptación del Artículo: 19. Mayo. 2011

Fecha de Revisión para publicación: 29. Noviembre. 2011 